\title{
Transformation of the Insurance Business Model using Block Chain Technologies
}

\author{
G. Venkatesh \\ Associate Professor (Marketing), Presidency Business School, Hebbal-Kempapura, Bangalore - 560024, Karnataka, \\ India; grenkatesh69@gmail.com
}

\begin{abstract}
The insurance sector has been less agile to adapt to rapid changes sweeping the external economic environment. However, massive advances in technology have now necessitated the insurance sector to relook at their business processes, archaic legacy systems and IT infrastructure to explore ways and means to serve the customers better. Block chain innovations in insurance are disruptive. Advent of block chain can mean simpler processes, lower premiums, greater transparency, increased accountability, increased efficiency and possibility of niche coverages. Block chain also enables smaller firms to cover risks that were earlier covered only by large insurance firms. However, there are limitations to implementing this technology due to the heavy investments involved in implementing block chain and due to change management challenges. There is scope for strategic alliances between insurance sector and insur-tech entrepreneurs to derive the maximum value out of block chain technology.
\end{abstract}

Keywords: Block Chain, Digital Ledger, Insurance, Innovations, Micro Insurance, Smart Contract

\section{Introduction}

Unforeseen and unfortunate circumstances necessitate an individual to seek insurance cover to protect his as well as his family's interests. Across the globe, the insurance sector has made a major contribution to a nation's economic growth. However, the insurance industry has been plagued with challenges like fragmented data sources, manual processing of claims, reliance on agents and brokers to serve clients, complex liability assessments for reinsurance, increasing instances of fraud, limited underwriting controls and inadequate exchange of information among key stakeholders. In the last few years, insurance mis-selling has been a major pain point for customers. Customers not adhering to the insurance principle of utmost good faith and providing incorrect information also cause challenges for insurers.

Today the insurance sector is facing the threat of competition from unexpected quarters-entrepreneurs who are using technology to scout new ways of underwriting risk and servicing customers in unique ways. While innovations by insurtech are classified as incremental innovations, block chain technologies are labelled as disruptive innovations. The ability of block chain to send, receive and store information can disrupt the way businesses process digital transactions.

Advent of block chain can mean simpler processes, lower premiums, greater transparency, increased accountability and possibility of niche coverages. Peer-to-peer insurance models and parametric models can be used to underwrite micro insurance covers. Block chain's security has proved to be its greatest strength.

Peer-to-Peer insurance is a risk sharing network where a group of individuals pool their premiums together to insure against a risk. This insurance is also called as social insurance. These platforms can begin using smart contracts to set claims and solve issues related to transfer of digital assets. In parametric insurance, insurers agree to

${ }^{*}$ Author for correspondence 
pay a certain sum upon the occurrence of triggers within preset smart contracts.

With the passage of time, technology has become simpler. Customers now evaluate an app within 1 minute. There is no room for technologies that don't appeal to customers because of their complexity. Apps like Ola, Uber, Zomato and Swiggy are so user friendly that even senior citizens find it easy to use.

From a single button interface to zero button interfaces- automation is becoming an integral part of customer journey.

\section{What is Block Chain?}

It has become the buzzword today. It is like a digital ledger. For example- when we buy or sell land, a ledger is the means by which two parties express the trust and confidence in an official manner. Block chain is a digital alternative for these ledgers. The annual spending on block chain has tripled since 2017 and is expected to reach nearly $16 \mathrm{~B} \$$ by 2023 according to $\mathrm{CB}$ Insights ${ }^{11}$.

Banking, ride sharing, messaging apps, hedge funding, internet advertising, education and academia, car leasing and sales, cloud storage, stock trading, real estate, health care, supply chain management, energy management, retail, 3D printing and manufacturing, publishing, food and beverage, air travel etc are other sectors where block chain shows lots of promise.

A block chain is a distributed, tamper-free, digital ledger. It is a global, online ledger that is accessible to everyone ${ }^{10}$. Block chain is now also termed as the "Internet of Contracts". Block chain has no owner- it is decentralized. Everyone can use Block chain as a basis for building future applications ${ }^{23}$. Data from the ledger is difficult to use for fraudulent purposes. Any change in the ledger gets immediately notified to others- irregularities run the risk of getting exposed due to the high degree of transparency involved in block chain (Figure 1).

Block chain is now being used to explore automation of contracts. A smart contract is a set of conditions recorded on the block chain so that claims can be paid out when the triggering event occurs ${ }^{8}$. Banks were needed for transfer of digital asset from one party to another party. Bitcoin has changed this. Now the transfer of digital assets does not need an intermediary. A decentralized network with thousands of computers (nodes) around the world controls the transfers. The transfer process can be automated leading to reduction in operating cost. This is how a smart contract operates. Smart contracts are lines of computer script that describe a scenario. Once this scenario is confirmed, contractual terms are implemented by Block chain. This is what forms the basis for insurance companies to tailor micro insurance contracts to unique needs of customers ${ }^{14}$.

All the participants in the network share the ledger - this is somewhat like everyone sharing a MS Excel document. Transactions are visible to all. Changes in the document get notified instantly. However, in block chain, a transaction once made can never be altered.

Customers get irritated when there is a delay in the transaction or there is a complexity that customer cannot relate to. Technology-enabled customer interfaces will arrest such service deficiencies leading to greater customer engagement and satisfaction. Block chains are expected to make customer transactions smooth and seamless. To manage the customer expectations, companies must make efforts in investing in new user interfaces ${ }^{14}$.

\section{Review of Literature}

Since 2008, the use of block chain technology has been steadily increasing. The benefits of security and data integrity add to its appeal (Yli-Huumo et al, 2016). The distributed digital ledger technology ensures transparency, traceability, and security (Saberi, Kouhizadeh, Sarkis and Shen, 2019).

Multiple processes between transacting parties characterize the insurance services. Transaction processing time, payment settlement time and security of the processes continue to remain concerns. Block chain is now being used by FinTech systems to address issues of security and productivity. Application of block chain in insurance will need an in-depth understanding of the underlying business processes in the insurance sector (Raikwar et al., 2018)

Despite holding such a promise, the real-world impact of this technology continues to be nebulous (Seebacher and Schuritz, 2017). Evolution of smart contracts can challenge traditional structures. Realizing the potential of block chain technology will need overcoming challenges 
of scalability, flexibility and use of external information (Hans et al., 2017).

Amidst the euphoria surrounding the usage and effectiveness of block chain technologies, it needs to be remembered that there is a risk in adopting this technology without an objective assessment of its added value. The insurance sector has not yet explored the complete spectrum of benefits that block chain can offer (Gatteschi, Lamberti et al., 2018).

\section{Need for Block Chain in Insurance}

Fragmented, old-world IT systems, siloed record keeping infrastructures, huge administrative costs and backend inefficiencies has prevented insurance companies from developing cost-effective insurance solutions for low income customers-particularly in the emerging economies of Asia. This ends up stunting the growth opportunities for the insurance sector.

Insurance business model is characterized by two critical processes-underwriting and claims settlement. These processes have become time-consuming and lack a system to accurately evaluate the information. Over the time, these processes have become slow, manual, paperbased, repetitive and prone to errors and duplication.

Block chain provides a unified platform rather than have a record keeping infrastructure that is siloed. It can eliminate the root cause of the inefficiencies that impact effective delivery of insurance services. As block chain operates through a decentralized platform that needs little central supervision, it is effective to fight fraud.

Block chain will play a disruptive role across the insurance value chain ${ }^{17}$. It can impact the processes like information collection, underwriting, actuarial analysis, billing, contract management, claims processing, distribution and policy administration. Regulators can access real time data. With same data available to all parties, the insurance platforms that are enabled by block chain technology can benefit from data sharing capabilities and back end analytics for pricing and risk. There can be a detailed audit trail of past insurance claims in order to detect fraud. The unconscionably long time required for onboarding and verification can be reduced.

\section{Micro Insurance and Block Chain}

Micro insurance is insurance coverage for poor people who subsist on low incomes. Micro insurance has suffered from challenges like high operational costs and ineffective distribution ecosystem. Let us consider that an insurance company has to underwrite a cover for protecting small farmers in developing countries from adverse conditions of weather that can affect yield of crops. Small farmers have land sizes that are small and so the cost of servicing such policies will be much more than the meagre premium earned through them.

Combinations of micro insurance and block chain are now being explored with an aim to make insurance as inclusive as possible 9 . The challenge is to make such micro insurance covers profitable for the insurance companiesotherwise they run the risk of not being sustainable over the long term.

Though the awareness about micro insurance is growing in India ${ }^{15}$, the Western world has managed to source technologies that can be integrated with service delivery processes so that costs can be optimized. Faster purchase of micro insurance policies and quicker resolution of claims becomes possible because the coverage is split into flexible and personalized products. Farm animals can be insured or even specific weather conditions can be insured. Claim values are smaller and automated processing reduces the cycle time to payout a claim. A chat bot that is enabled by Artificial Intelligence (AI) can help a customer choose the right products. Claims related information can be verified using AI and smart contracts can release the payments sans any sort of documentation.

In micro insurance, claims payments are triggered by a block chain enabled smart contract ${ }^{16}$.

The Indian government has subsidized crop insurance. Yet, the take-up rates for micro insurance in India are less than 5\%. Farmers do not trust the process as they don't believe that they will be paid when there is a loss of yield or prior experience would have sensitized them about the delay in the claims processing.

Parametric insurance covers can include natural disasters like earthquake, tropical cyclone and flood in their scope. As claims can be verified through automation, obviation of human intervention expedites the process. 


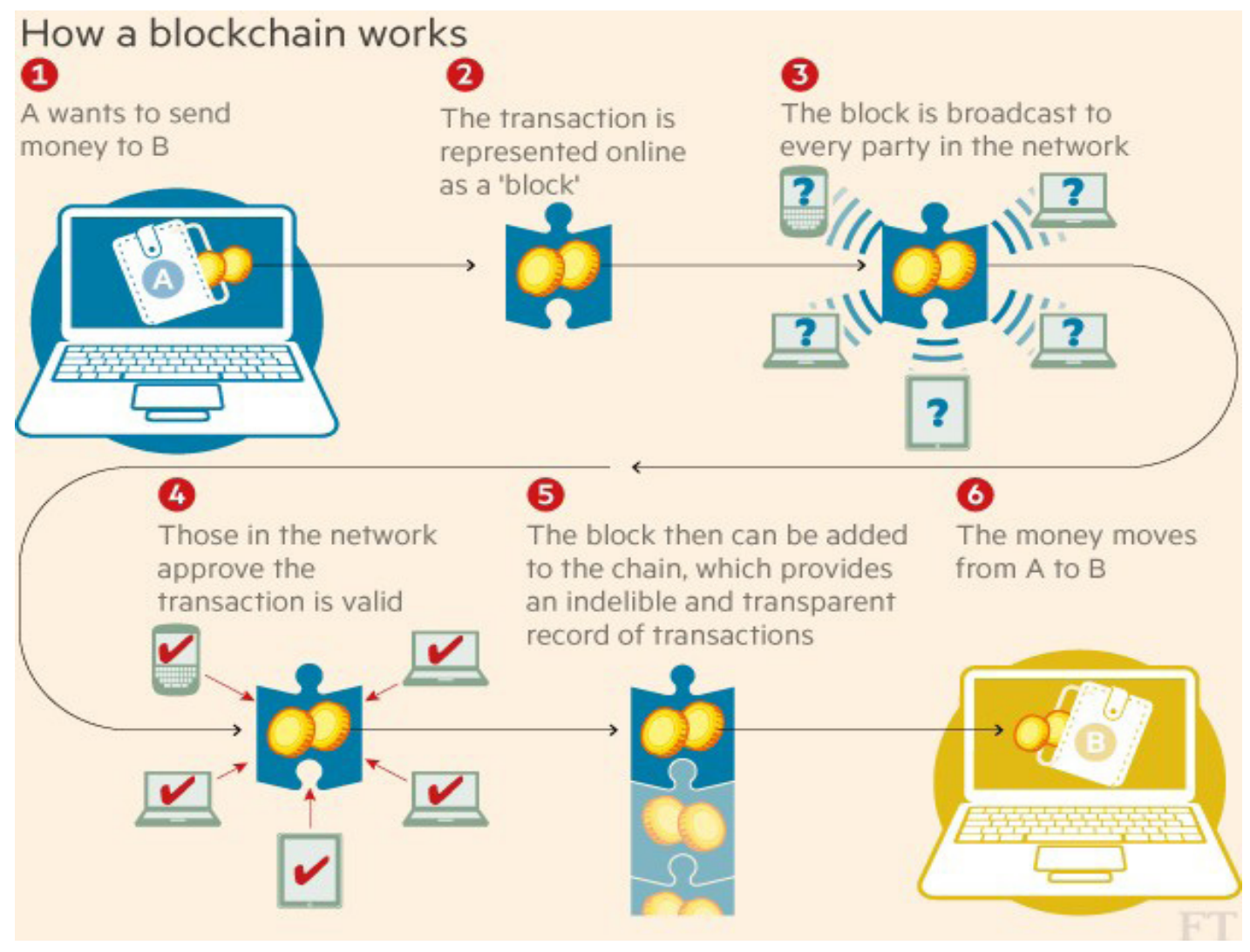

Figure 1. Source: https://www.weforum.org/agenda/2016/06/blockchain-explained-simply/

In India, delays in claims settlement have led to 95 million farmer households not being covered. Traditional insurance companies can take as long as one year to settle crop insurance claims. Insurer must send someone to assess the claim, there is an audit, the insurance company may submit a claim to a reinsurer...the process is elaborate.

A maize farmer in Ghana may be covered by a blockchain enabled smart contract. The farmer may become eligible for a claim if less than 200 millimeters of rainfall is observed during the growing season. Once the amount of rainfall is measured, a claim payment would be sent to the farmer via a mobile money service like M-PESA.

Lengthy proposal forms may not appeal to the marginalized sections. A personal face to face interaction with farmers and poorer sections of society is needed to explain the product features to them. When the smart contract is triggered, a text message will be sent to the farmer informing him about the payment details. One of the main challenges in block chain is to identify a trusted source for the trigger - especially when the locations are far flung.

Traditional insurance suffers from a trust deficit due to the inherent conflict of interest - insurance company can deny or delay the payout and benefit from the same. Smart contracts will reduce the trust deficit.

\section{Caselet ${ }^{13}$}

Etherisc is an insurtech company. It has demonstrated how block chain can lead to better cost management and enhanced efficiencies. The company introduced weather damage cover for Puerto Rico that was battered by a hurricane.

Etherisc has a technology-enabled platform. This locks the premiums in smart contracts on the public ethereum block chain. Pay outs are made when the triggering event 
occurs. For example - there could be a weather sensor that senses high-speed winds.

When such payouts are made, it spreads the word that insurance can be relied upon to mitigate the risks. This type of parametric insurance doesn't need the formal lodging of a claim. The insurer makes the payment on the occurrence of the triggering event. Use of a block chain reduces operating costs as the insurance company doesn't need too many employees for completing the transaction. Similar claims can be processed for covers to protect against flooding.

The success of these organisations in using block chain is a result of the external data source that would automatically inform the block chain whenever a triggering event occurs. AXA, an insurance giant, launched a block chain enabled parametric insurance contract for covering flight delays (example - if the plane is two hours late, the customer gets an automatic payout). AXA is now looking at extending such efforts for rural micro insurance. Traditional crop insurance schemes relied on measuring the yield as against the yield that was covered by insurance. Remote sensing technologies enable greater operational efficiency.

\section{Block Chain and its Disruptive Powers}

The insurance sector has been less agile to change in the past. This has increased the risk of cybercrimes and data breach.

Block chain is a revolutionary technology with power to transform the insurance business. Block chain can enable storage of numerous data and files. Transparent and traceable electronic transfer is possible due to block chain. Smart contracts are efficient because they minimize human intervention and use digital protocols where parameters are set up in advance. Blockchain can be applied efficiently and securely throughout the entire process of insurance underwriting, premium collection, indemnity payment and even reinsurance.

The conventional ways of the insurance industry -be it the purchasing process (through the agency model) or claims handling process-are proving to be irksome in an era where customer expectations have skyrocketed and customers are clamoring for greater value from insurers. The processes often assume a bureaucratic hue due to manual involvement at crucial stages. It is this lacuna that technologies like block chain are trying to address. The only solution appears to lie in use of artificial intelligence, smart contracts and automation.

There is increasing consensus about the disruptive power of block chain technology ${ }^{18,20}$. The World Bank, UNICEF and USAID feel that block chain can help the poorest of the poor.

Aon, Etherisc and Oxfam in Sri Lanka have collaborated to provide block chain enabled micro insurance to small holder farmers in Sri Lanka. The premiums are affordable for the paddy field rice farmers. The alliance has believed in empowering farmers. Low income households need a claims process that is simple, quick and can be easily understood. Automation makes data security and integrity a reality. Ideally when the focus of the business is deflected to support the marginalized sections of the society, sustainability of business is the collateral benefit.

\section{Impact of Block Chain on the Insurance Sector}

1. Event triggered smart contracts that enable automated claims will reduce fraud and lead to improved customer experience. The back-end support will be more efficient with reduced human error, less of data duplication and fewer processing delays. Transaction costs will be lowered. Smart contracts will lead to de centralized and fully digital markets that are safe. Manual intervention will be reduced.

2. The key members of the value chain will be connected through consortium models where seamless sharing of data becomes a distinct possibility ${ }^{21}$.

3. Real time underwriting will result in better pricing and risk assessment. Data sharing for pricing and analytics will be automatic. Huge volumes of data can be captured through sensors, IoT and trackers.

4. Greater penetration of underdeveloped insurance and micro insurance markets will be possible though distributed databases.

5. New types of insurance cover like sharing economy, travel insurance, spot insurance and hybrid policies will demand greater transparency 


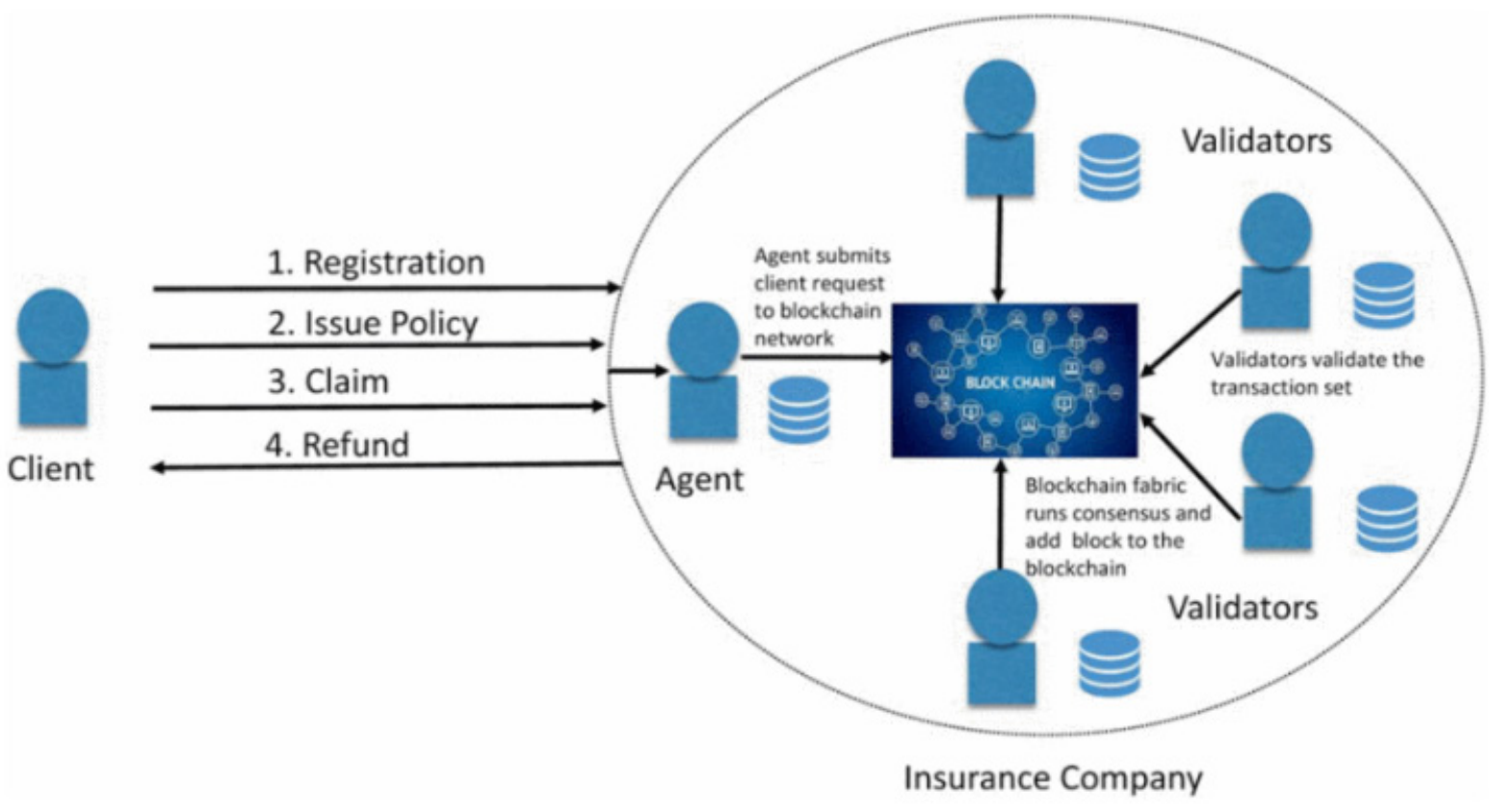

Figure 2. Source: innovate.ieee.org

and can be underwritten at significantly lower prices.

\section{Benefits of Block Chain in Insurance}

The distributed ledger technology can streamline the processing of insurance claims, improve cybersecurity protocols and minimize frauds. Smart contracts can replace manual processing of data. A smart contract executes based on certain conditions and agreement between the insurance company and policyholder. This can improve the speed of processing transactions (Figure 2).

In case of health insurance, insurers, insured and hospitals can be participants in the peer-to-peer communication network. Hospitals can make the medical history of a patient available to insurers and claims can be filed by policyholders on the same platform. Data once added cannot be modified by anyone. This improves transparency of the information shared.
A community whose members can collaborate and learn from one another is a pre-requisite for block chain. For example, in November 2016, an informal consortium of private life insurers was set up to share experiences and discuss how customer experiences can be improved and how levers can be set up for healthy growth of the insurance industry ${ }^{24}$.

Block chain can disrupt the way records are stored and secured. It will lead to increased collaboration among all the players. KYC data, health and policy records can be verified. Information management can be automated. Unclaimed life insurance ledger can be shared along with national policy and claims records. Even details of agents can be shared across the network.

Fraud can be minimized. Paperwork becomes more streamlined. Checks for agent recruitment and improving customer outreach for unclaimed amounts are possible due to block chain.

Decentralization can strengthen sharing of information among the various stakeholders and insurers must pay attention to pricing, product development, claims processing and management of their reputation risks. 
The tangible and intangible costs associated with adoption of block chain will be the biggest concern for insurers and reinsurers. The risks to existing insurance distribution business models as a result of implementation of block chain will need careful consideration. Block chains will need increased cyber security cover - again an added burden of investment.

\section{Challenges Specific to India}

The Indian insurance industry comprises both public and private players. Implementation of block chain-even if it is on a pilot scale-needs capital investment. Will the Indian insurers be ready to accept change? This is an important question. The industry has been accused of a laggard approach to respond to change. Private players are using digital technologies more than national insurance companies. However, the basic underlying principle of insurance-that of collecting premiums from several people to pay the claims of a few people is now being viewed with mistrust and suspicion. Though the industry waxes eloquent about customer service and the regulator is making attempts to streamline things, concerns remain for customers. Staking an insurance claim is a painful process for the customer. The industry that is so eager to market its products and services and collect premium adopts a stoic approach when it is a question of paying claims. Just because a few policyholders commit frauds-it cannot be surmised that every policyholder is fraudulent. The industry claims to offer protection but when a customer needs it the most, are the insurance processes agile enough to serve the customers in the best manner possible? This is a question that the industry in general and regulator (in particular) must strive to answer. Block chain technologies hold lots of promise and the Government must instigate the regulator to set up a think-tank to discuss the way forward.

\section{Conclusion}

Low growth rates, declining customer satisfaction and frequently recurring economic crisis are reasons why there needs to be a drastic transformation of insurance sector through disruptive technologies like block chain.

The insurance sector plays a crucial role in wealth creation and is an integral part of financial services industry. Governments must support the execution of strategies for increased penetration of insurance services and regulatory intervention is needed to achieve this goal. Any financial inclusion plan that doesn't include insurance services is incomplete ${ }^{12}$.

Block chain presents an exciting proposition because it enables smaller firms to cover risks that were usually covered by large insurance firms. Though it might be too early to predict if block chain technology will end up disrupting the traditional insurance market, the prospect of new players entering the market and using automation cannot be ruled out ${ }^{25}$.

Health and life insurers face increasing costs, discerning customers, and innovative disruption. It is worth exploring if block chain can enable them to cut costs, manage risks, improve customer service and bolster their bottom line.

Accurate pricing and better product design are positive outcomes of use of block chain. These platforms will also necessitate upgradation of industry processes. Therefore, the involvement of IRDA as a regulator deserves special mention.

Considering insurance is a business that has always thrived on intermediaries, the advent of block chain will streamline insurance processes through automation changing the very nature of the business model in the insurance industry.

It is expected that block chain will enable greater penetration of the micro insurance business. As claims processing begins after triggering of an event, there is no need for the policyholder to stake a claim. Companies are exploring ways in which weather index-based insurance products can be introduced in the market.

The irony is that even as the world population is increasing, land sizes are shrinking. Western nations are grappling with the food versus fuel debate when it comes to usage of land and enhancing farm productivity. Global warming is leading to vagaries in weather conditions. New insurance business models that rely on collaborative value chains can be expected in the future.

Insurtech firms are contemplating establishing smart contracts and risk pools with large conglomerates that have diversified business interests ${ }^{19}$. These pools may also include suppliers of farm equipments, farmers' co-operatives, donor organizations and government bodies. The main advantage of using block chain is that money will be paid out in line with what is stipulated in the smart contract. 
Personalized insurance covers that are affordable and effective are possible due to block chain technology. Industry experts opine that the insurance industry landscape will remain the same but the players will change in the next decade or so. Revamping of existing business model will be mandated by demands from the market. Insurers who take actions to implement innovative solutions will have greater staying power. Block chain can enable fraud prevention, track assets, reduce operational costs and enhance operational efficiencies.

It is expected that micro insurance, as an insurance vertical, will shoot into prominence due to the sheer volume of business and due to advances in block chain technology. Block chain can directly and indirectly improve insurance business models and processes. This in turn will enhance customer experience and improve operational effectiveness. As block chain is associated with security, there is greater trust among the participating entities. Block chain can collect all the relevant information and act on the data using smart contracts. Smart contracts can preclude fraudulent insurance claims ${ }^{22}$.

In health insurance, the application process for obtaining a cover as well as for filing a claim is nettlesome. Block chain can allow for easier access to a comprehensive set of medical records. Since health records are in an electronic form, the insured must be more vigilant in demonstrating the right behavior.

Block chain can reduce administrative costs by improving fragmented legacy IT systems. New security protocols and systems are needed for meeting customer expectations. In the absence of internal expertise on such emerging technologies it behooves that insurers have strategic alliances with experts in block chain development.

To realize blockchain's full potential as a business transformation opportunity, insurers will need to leverage several other technologies in tandem, including advanced analytics, artificial intelligence, and IoT, as well as collaborate with a wide range of stakeholders.

Leveraging the potential of block chain to transform the business model will need insurers to embrace other technologies like artificial intelligence and Internet of Things. This calls for formulation of standards to use block chain enabled data.

The available risk assessment tools and platforms are unable to offer custom solutions or innovative risksharing models. By using block chain solutions like smart contracts and decentralized payment networks, startups and insurtech firms seek to disrupt the insurance industry through the implementation of peer-to-peer and on-demand insurance solutions.

Block chain makes it easier for people to share risk within a smaller community and seek insurance for specific covers. Targeted insurance covers suited to specific consumer needs is what block chain is all about.

The picture that is now emerging is that block chain is going to upend insurance. Implementation of block chain must focus on standardization and consistency in approach. The future of insurance is all about intelligent adoption of Block chain. Smart contracts, digital currencies and fraud solutions will cease to be mere buzz words. Insurers must revamp their business models, underwriting processes as well as structure of the policy. Block chain technologies bring the customer into sharper focus. Strategic alliances between block chain start-ups, brokers, reinsurers, insurers may be a distinct possibility in the future. Successful implementation of block chain technologies in insurance will prove to be a game changer for the insurance industry.

The industry is under pressure to innovate and explore new sources of growth in Asian economies. A modernized regulatory framework complemented by adoption of block chain technology will open the opportunities for the insurance sector and drive economic growth.

\section{References}

1. Gatteschi, V., Lamberti, F., Demartini, C., Pranteda, C., \& Santamaría, V. (2018). Blockchain and smart contracts for insurance: Is the technology mature enough? Future Internet, 10(2), 20. https://doi.org/10.3390/fi10020020

2. Hans, R., Zuber, H., Rizk, A., \& Steinmetz, R. (2017). Blockchain and smart contracts: Disruptive technologies for the insurance market.

3. Mukherjee, Subhankar \& Pal, Parthapratim. (2017). Impediments to the Spread of Crop Insurance in India. Economic and Political Weekly, 52.

4. Raikwar, M., Mazumdar, S., Ruj, S., Gupta, S. S., Chattopadhyay, A., \& Lam, K. Y. (2018, February). A blockchain framework for insurance processes. IEEE: In 2018 9th IFIP International Conference on New Technologies, Mobility and Security (NTMS). pp. 1-4. https://doi. org/10.1109/NTMS.2018.8328731

5. Saberi, S., Kouhizadeh, M., Sarkis, J., \& Shen, L. (2019). Blockchain technology and its relationships to sustain- 
able supply chain management. International Journal of Production Research, 57(7), 2117-2135. https://doi.org/10 $.1080 / 00207543.2018 .1533261$

6. Seebacher, S., \& Schüritz, R. (2017, May). Blockchain technology as an enabler of service systems: A structured literature review. In International Conference on Exploring Services Science (pp. 12-23). Springer, Cham. https://doi. org/10.1007/978-3-319-56925-3_2

7. Yli-Huumo, J., Ko, D., Choi, S., Park, S., \& Smolander, K. (2016). Where is current research on blockchain technology? A systematic review. PloS one, 11(10), e0163477. https://doi.org/10.1371/journal.pone.0163477. PMid:27695049 PMCid:PMC5047482

8. Available from: https://minutehack.com/opinions/the-riseof-smart-contracts-using-blockchain

9. Available from: https://www.coindesk.com/inclusiveinsurance-businesses-see-blockchain-change-enabler

10. Available from: https://www.genre.com/knowledge/publications/iipc1808-1-en.html

11. Available from: https://www.cbinsights.com/research/ industries-disrupted-blockchain.

12. Available from: https://medium.com/impact-insurance/ the-opportunity-of-blockchain-technology-for-inclusiveinsurance-3e61733afd34

13. Available from: http://aon.mediaroom.com/newsreleases?item $=137772$

14. https://medium.com/theheartilab/micro-insurance-isinsurance-evolved-d834b33766d8
15. Available from: https://www.blockchain-council.org/ blockchain/how-blockchain-can-be-used-in-micro-insurance-how-it-works/

16. Available from: https://financialit.net/blog/micro-insurance-hasnt-worked-can-blockchain-fix-it

17. Available from: https://www.disruptordaily.com/blockchain-use-cases-insurance/

18. Available from: https://www.insureblocks.com/ep-9-blockchains-disruptive-potential/

19. Available from: https://www.eosventurepartners.com/ blockchain/

20. Available from: https://www.disruptordaily.com/blockchain-market-map-insurance/

21. Available from: https://www.expresscomputer.in/news/ indian-insurance-firms-come-together-to-form-informalconsortium-on-blockchain/20362/

22. Available from: https://techcrunch.com/2016/10/29/blockchain-is-empowering-the-future-of-insurance/

23. Available from: https://blockchainsimplified.com/blog/ application-of-blockchain-technology-in-insurance-sector/

24. Available from: https://www.sitepoint.com/blockchain-ininsurance-realizing-critical-growth-opportunities/

25. Available from: https://www2.deloitte.com/us/en/pages/ life-sciences-and-health-care/articles/blockchain-in-insurance.html 